\title{
Belphégor
}

\section{Maxime Prévost, Alexandre Dumas mythographe et mythologue. L'Aventure extérieure}

\section{Anthony Glinoer}

\section{(2) OpenEdition}

1 Journals

\section{Electronic version}

URL: https://journals.openedition.org/belphegor/1151

DOI: 10.4000/belphegor.1151

ISSN: 1499-7185

Publisher

LPCM

\section{Electronic reference}

Anthony Glinoer, "Maxime Prévost, Alexandre Dumas mythographe et mythologue. L'Aventure extérieure", Belphégor [Online], 16-1 | 2018, Online since 16 July 2018, connection on 19 October 2021. URL: http://journals.openedition.org/belphegor/1151; DOI: https://doi.org/10.4000/belphegor. 1151

This text was automatically generated on 19 October 2021

\section{(c) (i) (9)}

Belphégor est mis à disposition selon les termes de la Licence Creative Commons Attribution - Pas d'Utilisation Commerciale - Pas de Modification 4.0 International. 


\section{Maxime Prévost, Alexandre Dumas mythographe et mythologue. L'Aventure extérieure}

Anthony Glinoer

REFERENCES

Maxime Prévost, Alexandre Dumas mythographe et mythologue. L'Aventure extérieure, Paris, Honoré Champion, coll. « Romantisme et modernités », 2018. 
Alexandre Dumas

mythographe

et mythologue

L'Aventure extérieure

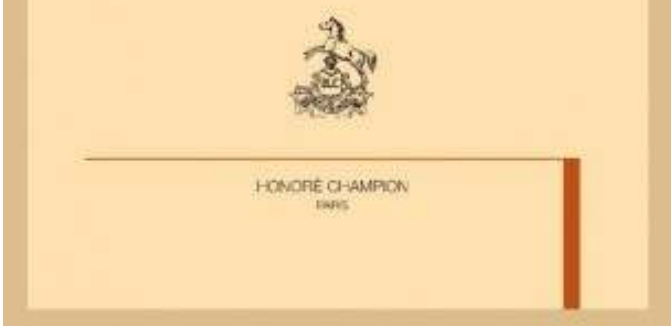

Dans ce livre, Maxime Prévost fait converger près de deux décennies de recherches, durant lesquelles il n'a cessé de proposer des rencontres aussi inattendues que fécondes : Jules Verne et Ian Fleming, Thomas de Quincey et Hubert Aquin, Beethoven et Victor Hugo ou encore Philippe Muray et Pierre Bourdieu. Ce livre n'est donc pas tout à fait un livre sur Alexandre Dumas père. Il est cela et plus que cela. Il y est question, en passant ou plus longuement, de Stevenson, de Flaubert, de Goscinny, de Conan Doyle ou encore de J. K. Rowling. Au cœur de l'entreprise réside une réflexion sur ce que Prévost appelle des " mythes laïcs ». Il entend par là non un récit mais un personnage, plus rarement un lieu (l'île au trésor par exemple) ou une donnée de l'intrigue (le voyage vers la lune), « dont la célébrité et le rayonnement transcendent ceux de leur créateur" (p. 30). Pour ne donner que quelques exemples de ces personnages devenus mythiques, à côté d'Athos et de Monte-Cristo dont il est abondamment question dans le livre, on peut mentionner Astérix, James Bond, Tintin, Gavroche, Cyrano de Bergerac, Dark Vador, etc. Seuls certaines productions fictives peuvent aspirer à ce statut d'œuvres mythiques (en ce sens qu'elles sont le creuset de personnages mythiques, devenus communs à toute une nation, voire à l'ensemble de la culture mondialisée). Ce sont ceux qui privilégient "l'expérience extérieure " par opposition à ceux qui, tels Flaubert ou Proust, ont plutôt tenté soit l'aventure intérieure, soit l'aventure formelle, voire les deux à la fois. Chez Prévost perce une certaine nostalgie du temps où la littérature essayait de guider les consciences avant que la littérature française la plus légitime, naturaliste et symboliste en particulier, ne rentre en dedans d'elle-même sous couvert de modernité. On pourra lui rétorquer qu'une Emma Bovary et son bovarysme ne jureraient pas dans le catalogue des personnages mythiques - témoins les nombreuses suites et transfictions (pour reprendre le concept développé par Richard Saint-Gelais) qui l'ont mise en scène et ont rejoué son destin. 
2 L'hypothèse sur laquelle repose l'édifice est forte : la pratique du roman-feuilleton a permis à « une poignée d'écrivains, que l'on qualifiera d'orchestrateurs de rejoindre, du jour au lendemain, la multitude - ou du moins ce qu'ils percevaient comme tel. » (p. 10) " Du jour au lendemain ", c'est-à-dire avec l'apparition de l'alliage entre la presse périodique diffusée à grande échelle et la fiction produite, entre autres, par quelques grands noms de tous connus : George Sand, Eugène Sue, Alexandre Dumas. L'œuvre et le personnage de Dumas servent donc à Maxime Prévost de laboratoire pour réfléchir à " l'impact concret de la littérature sur l'opinion publique et l'imaginaire social de la mi-siècle » (p. 11) et au-delà, précisément parce que Dumas a, avec Auguste Maquet, créé des mythes, c'est-à-dire des personnages qui ont acquis une notoriété durable et universelle, même auprès des non-lecteurs de littérature. Dumas est donc pris à témoin au nom des autre mages romantiques, ceux qui « avaient implicitement tenu pour acquis que l'art façonne tout le champ de la conscience, tout le domaine de l'intelligible, tout l'imaginaire social en somme, tant et si bien qu'il faille considérer la littérature comme une force historique vive. » (p. 236)

3 L'hypothèse est séduisante et la démonstration convaincante, même si je m'interroge sur le saut qui consiste à convertir la notoriété publique en ascendant sur le public. Maxime Prévost ne soutient pas, je le précise, que cet ascendant ait été concrètement observable, plutôt que si les personnages sont devenus durablement mythiques, si par exemple Arsène Lupin fait encore la joie d'enfants dans un manga animé japonais produit par Netflix, cela ne peut pas être seulement le symptôme d'une culture du recyclage et de la sérialité. C'est surtout parce que, dans la perspective de Jauss et de l'École de Constance, ces personnages et les situations dans lesquelles ils sont placés apportent des réponses à des questions qui ne sont pas toujours formulées, voire " dont on n'avait pas conscience qu'elle[s] se posai[en]t » (p. 55). Quelles sont ces questions ? chez Dumas, celle de l'honneur, celle de la noblesse, celle de l'héroïsme, celle de l'amitié et par extension de la communauté restreinte (au détriment de celle de l'amour, qui pourtant anime d'Artagnan pour Constance Bonacieux), celle du vieillissement du héros, etc. Autant de questions profondes que le roman mythique de " l'aventure extérieure ", malgré le discrédit dans lequel il est habituellement tenu, pose avec d'autant plus d'acuité qu'il les pose de génération en génération, d'un pays à l'autre. Tel est l'horizon de la démarche de Prévost, me semble-t-il : tirer Dumas jusqu'à nous (mais qui est ce " nous »?), lui rendre l'actualité des questions qu'il a soulevées. Pour mener sa démarche de façon si inductive, Prévost se sert de l'histoire culturelle mais pas seulement. Dans les réflexions théoriques qui surgissent au fil des pages, l'ancien membre du Collège de sociocritique de Montréal fait plutôt appel à la sociocritique de Claude Duchet et à la mythocritique de Gilbert Durand, appuyées sur les théories du mythe de Mircea Eliade et de Joseph Campbell notamment, en souhaitant l'avènement d'une " mythocritique historique » qui viendrait faire le pont entre histoire littéraire et histoire culturelle (p. 97).

4 Le propos est nettement divisé en deux parties qui s'enchaînement chronologiquement. Le premier temps, celui des Mousquetaires et du Comte de Monte-Cristo, voit un Alexandre Dumas créateur de mythes (le " mythographe ») ; le second, après les échecs des candidatures à la députation, est celui d'un Dumas devenu incapable de donner naissance à des personnages d'une telle ampleur et d'une telle célébrité, se propose plutôt de réfléchir aux mythes modernes (le " mythologue »). Dans la première partie, un chapitre est consacré au personnage de Monte-Cristo, personnage mythique par 
excellence, et un autre à la trilogie des mousquetaires et en particulier à leur "économie alcoolique ». On y voit un Dumas très politique, ayant la monarchie de Juillet en horreur, n'ayant jamais renoncé tout à fait à son admiration pour l'empereur et adhérant à une vision très méritocratique de la société. Dans la seconde partie, l'auteur se penche sur des œuvres, plus tardives et moins connues, et à des questionnements plus divers : les Mémoires d'un médecin et le paranormal, Les Mille et un fantômes et le fantastique, Isaac Laquedem et la tentation, parallèle à celle d'Eugène Sue avec ses Mystères du peuple, de la réécriture de l'histoire par le prisme d'un personnage immortel (ou de la descendance d'une famille dans le cas de Sue), Une odyssée en 1860 et l'écriture de l'histoire en marche, enfin les « causeries " publiées par Dumas dans les années 1860. Après s'être interrogé sur le succès du premier Dumas, Prévost questionne les échecs du second, notamment sur l'illisibilité de l'histoire de Joseph Balsamo, sur l'inachèvement de celle d'Isaac Laquedem et sur les insuffisances du fantastique dumasien en regard de la théorie du fantastique de Tzvetan Todorov.

5 Au bout du compte, tant les mises en scène de son scénario auctorial sont nombreuses et prégnantes chez lui, on en vient à se demander si Dumas n'a pas soigné plus qu'aucun autre son propre mythe : l'érection de la statue de son propre personnage d'écrivain. Et avec quelque succès. Après tout, après avoir incarné Monte-Cristo et Porthos (mais aussi Jean Valjean), Gérard Depardieu n'en est-il pas venu à jouer Dumas le panthéonisé lui-même dans le film L'autre Dumas (2010)?

\section{AUTHOR}

\section{ANTHONY GLINOER}

Chaire de recherche du Canada sur l'histoire de l'édition et la sociologie du littéraire Université de Sherbrooke 